\title{
A model for the optimization of beacon message age-of-information in a VANET
}

\author{
Andrea Baiocchi and Ion Turcanu \\ Dept. of Information Engineering, Electronics and Telecommunications (DIET) \\ University of Roma Sapienza - Via Eudossiana 18, 00184 Roma, Italy \\ \{andrea.baiocchi, ion.turcanu\}@uniromal.it
}

\begin{abstract}
Beaconing is a basic communication process taking place in Vehicular Ad Hoc Networks (VANETs) to achieve cooperative awareness among vehicles on the road. It is actually a paradigm of information spreading among peer-agents, where each node of a networks sends periodically broadcast messages containing information collected by the node itself. A trade-off arises between the update frequency of the broadcast information and the congestion induced in the wireless shared channel used to send the messages, which is based on the IEEE 802.11p standard in case of a VANET. For periodic updates, the primary metric is the Age-of-Information (AoI), i.e., the age of the latest update received by neighboring nodes. We define an analytical model to evaluate the AoI of a VANET, given the connectivity graph of the vehicles. Analytical results are compared to simulation to assess the accuracy of the model. The model provides a handy tool to optimize the AoI trade-off.
\end{abstract}

\section{INTRODUCTION}

A common messaging paradigm in the context of the Internet of Things (IoT) consists of a network of peer-agents sending broadcast update messages to one another. These messages carry state variables of sending nodes and/or information collected locally by sending nodes, which is spread to their neighbors. Each node maintains a map of the latest updates or possibly of the most updated processing results obtained from the stream of data it receives continually from its neighbors.

A specific major example of this communication paradigm is a Vehicular Ad Hoc Network (VANET), where vehicle nodes exchange beacon messages to maintain cooperative awareness of one another. In this context the Dedicated ShortRange Communication (DSRC) standard [1] has been defined, based on IEEE 802.11p PHY and MAC layers, fully compliant with ETSI ITS-G5 [2] standard. On top of this CSMA-based communication technology, safety and traffic information or efficiency applications have been defined, such as Cooperative Awareness Messages (CAMs) [3] and Decentralized Environmental Notification Messages (DENMs) [4]. The former ones are sent out periodically by each equipped vehicle to inform about its type, position, direction of movement, speed and other optional features. The latter ones are intended to provide early warning of potentially critical road and traffic events, aiming ultimately to enhance travel safety.

Examples of message sending in different contexts include wireless sensor networks [5] (of whom the VANET is one major, special example), gossiping algorithms [6], [7], distributed consensus algorithms [8], [9], network of automata [10], and synchronization of coupled nodes through a network [11]. The common point of all these contexts is that some kind of dynamical process evolves over a network, possibly large and time-varying, formed by a set of peer-agents. The evolution of the process is tied to message passing and state updates among nodes. Most of those examples need periodically updated information on a time scale compatible with the application requirements.

A key point in the exchange of cooperative messages, as well as in general in the broadcast of update messages to neighbors in any network of distributed agents communicating through a shared channel, is to control the congestion level so as not to impair the regular and timely update of time-critical information. Reducing the sending rate of updating messages is the obvious control variable to avoid congestion, yet it leads to a smaller refresh rate of information. The trade-off between congestion of the communication channel and refresh rate of the information carried by the messages has brought to the definition of a specific metric, the Age-of-Information (AoI). Given a table at node $i$ whose entries are updates from another node $j$, the AoI at time $t$ of those updates is measured by $A_{i j}(t)=t-u_{k}(j \rightarrow i)$ for $t \in\left[u_{k}(j \rightarrow i), u_{k+1}(j \rightarrow i)\right)$, where $u_{k}(j \rightarrow i)$ is the time when the $k$-th update from $j$ is received by $i$. Whenever a new update is received, the AoI drops to zero; then it grows by 1 second per second of elapsed time until the next update. A sample path of the AoI metric is shown in Figure 1.

The shared wireless channel is run typically according to a random access protocol, given that usually no central coordination exists and resource partitioning and allocation would entail a lot of signaling, hence inducing an unacceptable overhead with respect to the target of the resource allocation (i.e., sending relatively short messages, often ranging between few hundred to few thousand bytes). With random access, as the level of contention for the medium grows because of the increasing activity of the nodes, collisions bring to message losses. Since broadcast mode is used in the application considered in this work, no acknowledgement and retransmission scheme is used at MAC layer. The message loss due to a collision is recovered when an update message is eventually received. Obviously, message losses impact negatively on the AoI.

AoI has been addressed specifically in [12], [13], [14]. Kaul et al. [12] gives a nice general model to evaluate the AoI of a population of information sources coupled to a central server 


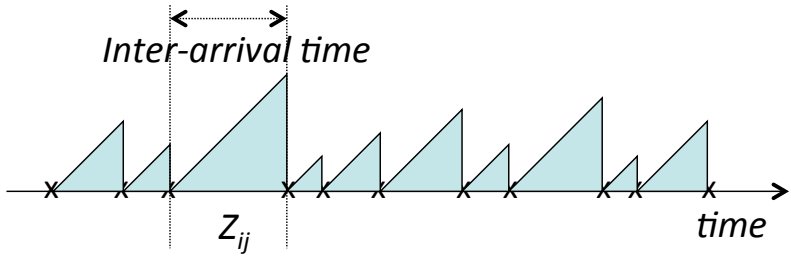

Figure 1. A sample path of AoI.

by means of a limited capacity channel. The general abstract model is able to highlight the basic trade-off involved in the AoI. Yet it refers to a single shared channel, not a distributed network of interacting agents. Kaul et al. [13] devise an adaptive beaconing strategy to minimize the age of information by balancing the load (hence the contention level) on the access wireless network and the frequency of updates. The addressed scenario is a vehicular network. Franco et al. [14] address the design of a cross-layer MAC protocol optimized to reduce the age of information in WLAN with high level of contention.

As for CSMA network modeling, since the classic model of IEEE 802.11 CSMA/CA of Bianchi [15], several extensions have been defined. Liew et al. [16] gives a relatively simple model to evaluate the performance of CSMA networks with partial sensing. The model addresses saturated nodes. Besides, it implies the computation of Maximum Independent Sets (MISs) of the network graph, where an arc is introduced between nodes $i$ and $j$ if both $i$ and $j$ can hear each other. This computation becomes impractical for graphs with more than several hundred links. A general CSMA network model is presented in [17] to address both saturated and non saturated CSMA/CA networks with partial sensing. The analysis is elegant and extremely powerful. However, the continuous-time model defined in that work does not account for collisions. Moreover, it brings about the same unfeasibility problem as with [16], since it requires the computation of the MISs of the network graph.

The contribution of this work is twofold. On a methodological level, we aim at stating and assessing an analytical model that can capture the distributed contention in a CSMA network to calculate the AoI. On the application level, we investigate how the key parameters of the MAC layer and of the messaging protocol impact on the AoI in a VANET, by considering a realistic urban scenario describing the vehicular traffic within the city of Cologne (Germany), as well as an artificially created Manhattan Grid scenario, based on artificial traffic generation and communication.

In the following, the system scenario for the VANET application is layed out (Section II). Then, the analytical model is developed (Section III). The simulation setup for the assessment of the model accuracy is introduced in Section IV. Performance results are displayed in Section V. Concluding remarks are given in Section VI.

\section{SYSTEM SCENARIO FOR THE VANET APPLICATION}

In this article we consider a particular example of wireless sensor networks, namely VANETs, where the entities exchanging the information are represented by vehicles roaming in an area of interest. This is a typical example of a dynamic and evolving network, with the state and the topology continuously changing over time. In such a scenario, vehicles are considered as mobile network nodes able to communicate among each other using the DSRC technology.

Timely and updated information is essential for a wide range of applications, varying from safety to urban sensing in general. Most of these applications rely on cooperative awareness, which can be achieved through regular exchange of messages containing application specific information. For instance, vehicles' position, dynamics and attributes have to be exchanged to support safety related applications. Vehicles can also act as mobile sensing platforms, meaning that environmental information can be periodically exchanged for urban sensing purposes. All this data needs to be packed up and delivered to every vehicle with a certain frequency to guarantee the freshness of the information (i.e., the AoI) required by each specific application.

As an example, the European Telecommunications Standards Institute (ETSI) defined two types of such messages: CAMs [3] and DENMs [4]. CAMs are periodically exchanged messages operated by the cooperative awareness basic service in the ITS G5 facility layer. The information contained in this type of messages is generally related to the vehicle status (e.g., position, speed, time, etc.) and attributes (e.g., vehicle type, dimensions, etc.). The generation period of a CAM, that is, the time interval between two consecutive CAM generations, may vary and depends on different factors, like the vehicle current status or the radio channel load. However, the ETSI ITS G5 standard defines a lower and upper bound for this generation period, which is $100 \mathrm{~ms} \leq T_{\mathrm{msg}} \leq 1000 \mathrm{~ms}$.

DENMs are event-based messages, which are triggered whenever an exceptional event occurs and vehicles roaming in the near geographic area have to be informed. The content of these messages is related to the type, position and duration of the incident. A vehicle detecting an unusual event, starts broadcasting DENM messages, which can be retransmitted by other receiving vehicles, for the whole time this event is present. Relevant information from both CAM and DENM messages is maintained in a local data structure, named Local Dynamic Map (LDM) [18]. All interested applications have access and rely on the updated information contained in these LDMs. The LDM is updated every time a new CAM or DENM is received and the content of this message is fresher than the information currently present in the LDM. If the CAMs or DENMs are lost due to congestion, collisions, etc., then the information inside the LDM becomes outdated, which may affect time-critical applications. The AoI metric is defined to measure and quantify how old is the information contained in the LDM at any point in time.

The considered scenario consists of an urban Region of Interest (RoI) defined by the road map where vehicles move. Vehicles are equipped with On-Board Units (OBUs) and transmit periodically broadcast messages. Each vehicle maintains a database of the last update received by neighboring vehicles. New records are added as updates are received, while stale 

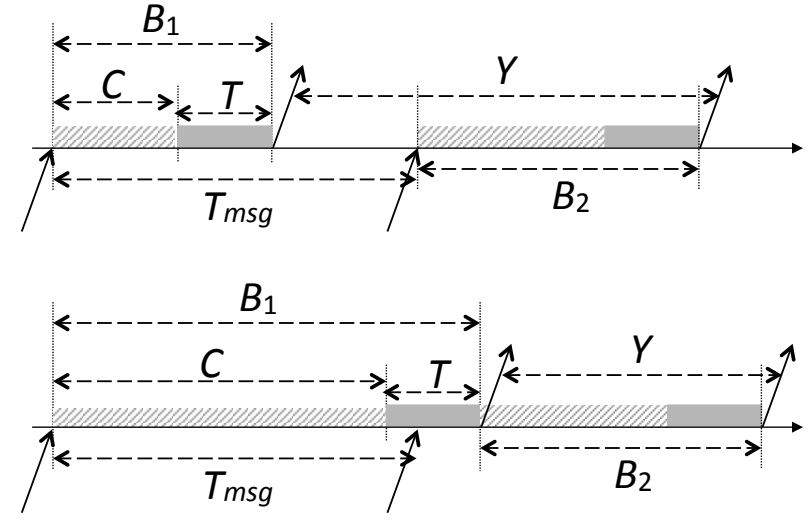

Figure 2. A sample path of AoI.

information is purged out of the table after a timer expires. Details of the simulation model are given in Section IV.

\section{ANALYTICAL MODEL FOR AOI EVAlUATION}

We focus on periodic one-hop message exchange, with nodes sending messages of given length $L$ with period $T_{\mathrm{msg}}$. New messages are accepted by the MAC layer entity of a node as long as it is idle. If the node is busy, the arriving new message is stored in a buffer. Further arriving messages are overwritten so that only the latest message is taken care of by the MAC layer, as soon as the previous message has been sent out on the channel. This setting is consistent with the periodic issuing of the beacon messages, each carrying an update of the vehicle information. Given this setting, only the latest update is worth being transmitted.

The model describes the generic node operation with a renewal process. Let us consider a tagged node $A$ sending a message at time $t_{k}, k \in \mathbb{Z}$. We let $Y_{k}=t_{k}-t_{k-1}$. At equilibrium, we can assume $Y_{k} \sim Y$. Since the times $\left\{t_{k}\right\}_{k \in \mathbb{Z}}$ are regeneration points for the sending process, the sequence of intervals $\left\{Y_{k}\right\}$ forms a renewal process ${ }^{1}$. We can distinguish two cases according to whether the contention plus transmission time is less than the message inter-arrival time (top picture in Figure 2) or not (bottom plot on Figure 2).

Then

$$
Y=B_{2}+\max \left\{0, T_{\mathrm{msg}}-B_{1}\right\}
$$

where $B$ is a random variable defined as the time elapsing from the moment when the MAC layer takes in charge a PDU until it eventually sends it out on the radio channel.

$B$ is the sum of the transmission time $T$ (including the overhead) and of the time $C$ spent on counting until the backoff counter hits 0 . If the channel is sensed busy, the counter is frozen until the channel activity terminates. Otherwise, the counter is decremented after a back-off time slot of duration $\delta$. Let $C$ denote the count down time, defined as the sum of a number of "slot" times, each slot lasting either $\delta$, the IEEE $802.11 \mathrm{p}$ back-off slot duration, or $T$, which is the time required

\footnotetext{
${ }^{1}$ At least, this is true under the simplifying assumption of independence of the stations' states.
}

to complete a MAC PDU transmission, including PHY and MAC overhead, and the ensuing DIFS ${ }^{2}$. Then

$$
C=\sum_{j=1}^{N} X(j)
$$

where $N$ is a discrete random variable with uniform distribution over $\left[0, W_{0}-1\right], W_{0}$ being the base contention window size of IEEE $802.11 \mathrm{p}$, and $X(j)$ are i.i.d. random variables with the same distribution as $X$ defined by

$$
X= \begin{cases}\delta & w \cdot p \cdot 1-b \\ T & w \cdot p . b\end{cases}
$$

Here $b$ is the probability that the tagged node senses the channel busy.

In the evaluation of the statistics of $X$ and hence of $C$, we must account for the fact that only partial sensing is realized in general. In other words, while some neighbor $N_{1}$ of the tagged node $A$ is transmitting, and hence the tagged node freezes its count-down state, some other neighbor $N_{2}$ of $A$ could start its own transmission, in case $N_{2}$ is out of carrier sensing range of $N_{1}$, i.e., $N_{1}$ is hidden to $N_{2}$. The resulting effect as seen by the tagged node $A$ is that its freezing time lasts more than $T$. This 'expanded' duration of the activity sensed on the channel by $A$ depends on the maximum number of nodes that can start transmitting independently of one another, i.e., that do not sense one another. Let $n_{\text {MIS }}$ denote the cardinality of the MIS around $A$. Once the transmission starts, up to $n_{\text {MIS }}-1$ more transmissions could start with random phases. By assuming independence and uniform probability distribution of the relative phasing in $[0, T]$, it can be found easily that the time $T$ is replaced by $T\left(2-1 / n_{\mathrm{MIS}}\right)$. The 'expansion' factor $\psi=2-1 / n_{\mathrm{MIS}} \geq 1$ of the activity time reduces to 1 when $n_{\text {MIS }}=1$, i.e., all neighbors of $A$ do sense each other. A proxy of the number $n_{\text {MIS }}$ that is easier to calculate is $\hat{n}=c+\nu(1-c)$, where $\nu$ is the number of neighbors of $A$ and $c$ is the clustering coefficient of $A$. This is simply a linear interpolation between $n_{\mathrm{MIS}}=1$ when $c=1$ and $n_{\mathrm{MIS}}=\nu$ when $c=0$. The clustering coefficient of a graph node $A$ is the ratio of the number of links among the $\nu$ neighbors of $A$ divided by the maximum number of such links, i.e., $\nu(\nu-1) / 2$. Given the adjacency matrix $\mathbf{A}$ of an undirected graph, the clustering coefficient of node $i$ is $c_{i}=\ell_{i} /[\nu(\nu-1) / 2]$, where $\ell_{i}$ can be found as the $i$-th element of the diagonal of the matrix $\mathbf{A}^{3} / 2$.

In the definition of $X_{i}$, the random variable $X$ at node $i$, we therefore substitute $T$ with $T \hat{\psi}_{i}$, where $\hat{\psi}_{i}=2-1 / \hat{n}_{i}=$ $2-\frac{1}{c_{i}+\nu_{i}\left(1-c_{i}\right)}$, with $c_{i}=\frac{2 \ell_{i}}{\nu_{i}\left(\nu_{i}-1\right)}$.

We have the following identities for the first two moments of $B_{i}=T+C_{i}$

$$
\begin{aligned}
& \mathrm{E}\left[B_{i}\right]=T+\frac{W_{0}-1}{2} \mathrm{E}\left[X_{i}\right] \\
& \sigma_{B_{i}}^{2}=\frac{W_{0}^{2}-1}{12}\left(\mathrm{E}\left[X_{i}\right]\right)^{2}+\frac{W_{0}-1}{2} \sigma_{X_{i}}^{2}
\end{aligned}
$$

${ }^{2}$ SIFS and ACK times are not included, since MAC PDUs for beaconing are sent in broadcast, hence no ACK is provided. 
where

$$
\begin{aligned}
& \mathrm{E}\left[X_{i}\right]=\delta\left(1-b_{i}\right)+T \hat{\psi}_{i} b_{i} \\
& \sigma_{X_{i}}^{2}=\left(T \hat{\psi}_{i}-\delta\right)^{2} b_{i}\left(1-b_{i}\right)
\end{aligned}
$$

The first two moments of $Y_{i}$ are found by considering all realizations $\beta_{k}$ of the random variable $B_{i}$ and the relevant probabilities, i.e., $p_{i}(k) \equiv \mathcal{P}\left(B_{i}=\beta_{k}\right)$. By definition:

$\mathrm{E}\left[\left(\max \left\{0, T_{\mathrm{mgg}}-B_{i}\right\}\right)^{\gamma}\right]=\sum_{k=0}^{W_{0}-1} p_{i}(k)\left(\max \left\{0, T_{\mathrm{mgg}}-\beta_{k}\right\}\right)^{\gamma}$

for $\gamma \geq 1$, and then Equation (1) yields

$$
\begin{aligned}
& \mathrm{E}\left[Y_{i}\right]=\mathrm{E}\left[B_{i}\right]+\mathrm{E}\left[\max \left\{0, T_{\mathrm{msg}}-B_{i}\right\}\right] \\
& \operatorname{Var}\left(Y_{i}\right)=\operatorname{Var}\left(B_{i}\right)+\operatorname{Var}\left(\max \left\{0, T_{\mathrm{msg}}-B_{i}\right\}\right)
\end{aligned}
$$

where, for $k=0, \ldots, W_{0}-1$, we have

$$
p_{i}(k)=\mathcal{P}\left(B_{i}=\beta_{k}\right)=\frac{1}{W_{0}} \sum_{m=0}^{W_{0}-1-k}\left(\begin{array}{c}
m+k \\
k
\end{array}\right) b_{i}^{k}\left(1-b_{i}\right)^{m}
$$

and

$$
\beta_{k}=T+W_{0} \delta+k\left(T \hat{\psi}_{i}-\delta\right)
$$

The probability that the $i$-th node attempts a transmission on the channel is

$$
\tau_{i}=\tau_{0} \frac{\mathrm{E}\left[B_{i}\right]}{\mathrm{E}\left[Y_{i}\right]}
$$

where $\tau_{0}$ is the probability of attempting a transmission in a saturated CSMA/CA network, when binary exponential backoff is not used and only the basic contention window size is used. Hence, $\tau_{0}=2 /\left(1+W_{0}\right)$, with $W_{0}=15$, according to the IEEE $802.11 \mathrm{p}$ standard. Note that nodes do not operate necessarily in saturation, since they are requested to send one message every $T_{\mathrm{msg}}$. As long as $B_{i}<T_{\mathrm{msg}}$ node $i$ completes contention and message transmission before the next message is ready to send. This is the typical case for standard message periods (between $100 \mathrm{~ms}$ and $1000 \mathrm{~ms}$ ), given that the contention time ranges between few ms and several tens of ms typically.

There remains to characterize the probability $b$. Let us introduce a subscript $i$ for the tagged node. Let $a_{i j}$ denote the entry $(i, j)$ of the adjacency matrix $\mathbf{A}$ of the carrier sensing graph of the nodes. In words, $a_{i j}=1$ if and only if node $j$ can receive (detect) the signal emitted by node $i$. Since the radio channel is reciprocal, we can assume that $\mathbf{A}$ is symmetric. In this model, we assume that the carrier sensing matrix $\mathbf{A}$ is given (see Section V).

As $\tau_{j}$ is the probability that node $j$ is found transmitting, the probability that a neighbor node $j$ of $i$ is not transmitting is $1-\tau_{j} a_{j i}$. We adopt the common independence assumption, whereby the states of the competing nodes in the CSMA network are assumed to be independent of one another. Then, the probability that node $i$ senses an idle channel, i.e., that all its neighbors are silent, is ${ }^{3}$

$$
1-b_{i}=\prod_{j=1}^{n}\left(1-\tau_{j} a_{j i}\right)
$$

\footnotetext{
${ }^{3}$ Note that we define $a_{i i}=0$.
}

where $n$ is the number of nodes in the network, hence the size of the adjacency matrix.

Summing up, the $\tau_{i}$ 's can be found by solving a system of non-linear equations made up of Equations (3), (6) and (7). If we write $\tau \equiv\left[\begin{array}{llll}\tau_{1} & \tau_{2} & \ldots & \tau_{n}\end{array}\right]$, the equation system can be written in a compact form as $\tau=\mathbf{F}(\tau)$. The function $\mathbf{F}(\cdot)$ is continuous and maps the unit hypercube into itself. Hence, Brouwer's theorem guarantees that there exists a fixed point.

Once the transmission probabilities $\tau_{i}$ are computed, we can find the conditional probability of success, $P_{s}(i, j)$, of the event that node $j$ receives a message from node $i$, given that $i$ transmits the message. This amounts to node $i$ transmitting and: (i) none of the neighbors of $j$ being active at the same time; (ii) node $j$ not transmitting as well. We can divide the neighbors of $j$ into two sets:

$\mathcal{A}_{i, j}$ the set of neighbors of $j$ that are also neighbors of $i$; $\mathcal{B}_{i, j}$ the set of neighbors of $j$ that are not neighbors of $i$.

The nodes belonging to the first set are synchronized by the activity of $i$, while the other nodes are not. Therefore, the transmission probability for node $k \in \mathcal{A}_{i, j}$ is $\tau_{k}$. Nodes in $\mathcal{B}_{i, j}$ are outside the communication range of $i$, hence they are hidden with respect to $i$. We assume they are completely desynchronized with $i$, hence node $k \in \mathcal{B}_{i, j}$ can start transmitting in any slot time of duration $\delta$ with probability $\delta / \mathrm{E}\left[Y_{k}\right]$. The vulnerability interval of the message sent by node $i$ to node $j$ comprises $m \equiv 2 T / \delta-1$ slot times. Therefore

$$
P_{s}(i, j)=\left(1-\tau_{j}\right) \prod_{k \in \mathcal{A}_{i, j}}\left(1-\tau_{k} a_{k j}\right) \prod_{k \in \mathcal{B}_{i, j}}\left(1-\frac{\delta}{\mathrm{E}\left[Y_{k}\right]} a_{k j}\right)^{m}
$$

for all $j \neq i$. The time $Z_{i j}$ to deliver a new message from $i$ to $j$ is given by

$$
Z_{i j}=\sum_{r=1}^{N_{i j}} Y_{i}(r)
$$

where $Y_{i}(r) \sim Y_{i}$ are the times between successive transmission attempts of node $i, Y_{i}$ is given in Equation (1), and $N_{i j}$ is the number of attempts required to make a successful message transfer from $i$ to $j$. Assuming that successive attempt outcomes are independent of one another, $N_{i j}$ has a geometric probability distribution, i.e.

$$
\mathcal{P}\left(N_{i j}=h\right)=P_{s}(i, j)\left[1-P_{s}(i, j)\right]^{h-1}
$$

for $h \geq 1$. The AoI at node $j$ for messages coming from $i$ equals $t-t_{i j}(k)$ for $t \in\left[t_{i j}(k), t_{i j}(k)+Z_{i j}\right)$, where $t_{i j}(k)$ is the time of arrival of the $k$-th message from $i$ to $j$.

The mean value of the AoI from $i$ to $j, H_{i j}$, is akin to the mean remaining service time in a queue, i.e.

$$
\mathrm{E}\left[H_{i j}\right]=\frac{\mathrm{E}\left[Z_{i j}^{2}\right]}{2 \mathrm{E}\left[Z_{i j}\right]}
$$


It is

$$
\begin{aligned}
\mathrm{E}\left[Z_{i j}^{2}\right] & =\mathrm{E}\left[N_{i j}\left(N_{i j}-1\right)\right]\left(\mathrm{E}\left[Y_{i}\right]\right)^{2}+\mathrm{E}\left[N_{i j}\right] \mathrm{E}\left[Y_{i}^{2}\right] \\
& =\frac{2\left[1-P_{s}(i, j)\right]}{P_{s}(i, j)^{2}}\left(\mathrm{E}\left[Y_{i}\right]\right)^{2}+\frac{1}{P_{s}(i, j)} \mathrm{E}\left[Y_{i}^{2}\right] \\
& =\frac{2-P_{s}(i, j)}{P_{s}(i, j)^{2}}\left(\mathrm{E}\left[Y_{i}\right]\right)^{2}+\frac{1}{P_{s}(i, j)} \sigma_{Y_{i}}^{2} \\
\mathrm{E}\left[Z_{i j}\right] & =\frac{1}{P_{s}(i, j)} \mathrm{E}\left[Y_{i}\right]
\end{aligned}
$$

The expressions above allow to compute the mean AoI of messages flowing from $i$ to $j$. The AoI at $j$ can be obtained by averaging over all neighbor nodes of $j$, if any. If $j$ is isolated, it receives no message actually, so AoI is meaningless. Besides this marginal case, we can define

$$
\mathrm{E}\left[H_{j}\right]=\frac{\sum_{i=1}^{n} a_{i j} \mathrm{E}\left[H_{i j}\right]}{\sum_{i=1}^{n} a_{i j}}
$$

provide that $\nu_{j} \equiv \sum_{i=1}^{n} a_{i j}>0$. The overall average AoI of the entire network can be summarized by the following definition:

$$
\mathrm{E}[H]=\sum_{j=1}^{n} \frac{\nu_{j}}{\nu} \mathrm{E}\left[H_{j}\right]=\frac{1}{\nu} \sum_{j=1}^{n} \sum_{i=1}^{n} a_{i j} \mathrm{E}\left[H_{i j}\right]
$$

where $\nu=\nu_{1}+\cdots+\nu_{n}=\sum_{j=1}^{n} \sum_{i=1}^{n} a_{i j}$.

\section{Simulation MODEL}

To validate the proposed analytical model, we compare its performance with a realistic simulation model of a VANET. The main roles of the simulations set is to test the key simplifying assumptions of the analytical model, namely:

- independence of the node states, used to derive the message delivery success probabilities;

- vehicle mobility, not accounted for in the analytical model;

- details of the MAC protocol in a partial sensing environment.

In particular, we consider a set of two simulation scenarios. The first one is an artificially generated Manhattan Grid scenario, created using realistic road lengths and building dimensions taken from downtown Manhattan. This scenario consists of vertical roads representing main avenues, each road having a total of 4 lanes (2 lanes per direction), and of horizontal roads representing secondary streets, each street having a total of 2 lanes (one lane per direction). The distance between 2 junctions on the horizontal and vertical roads is of 275 and $80 \mathrm{~m}$ respectively. Parallel roads are separated by buildings obstructing the inter-vehicle DSRCbased communication. Both vehicular mobility and networking are simulated over a larger area, but the observed region is smaller in order to avoid border effects. In particular, the target area is enclosed in a $620 \mathrm{~m} \times 530 \mathrm{~m}$ region situated in the center of our simulated scenario. This area contains 3 vertical and 7 horizontal roads as described above.

The second simulation scenario that we consider is based on the TapasCologne ${ }^{4}$ [19] vehicular mobility dataset (see

\footnotetext{
${ }^{4}$ http://kolntrace.project.citi-lab.fr/
}

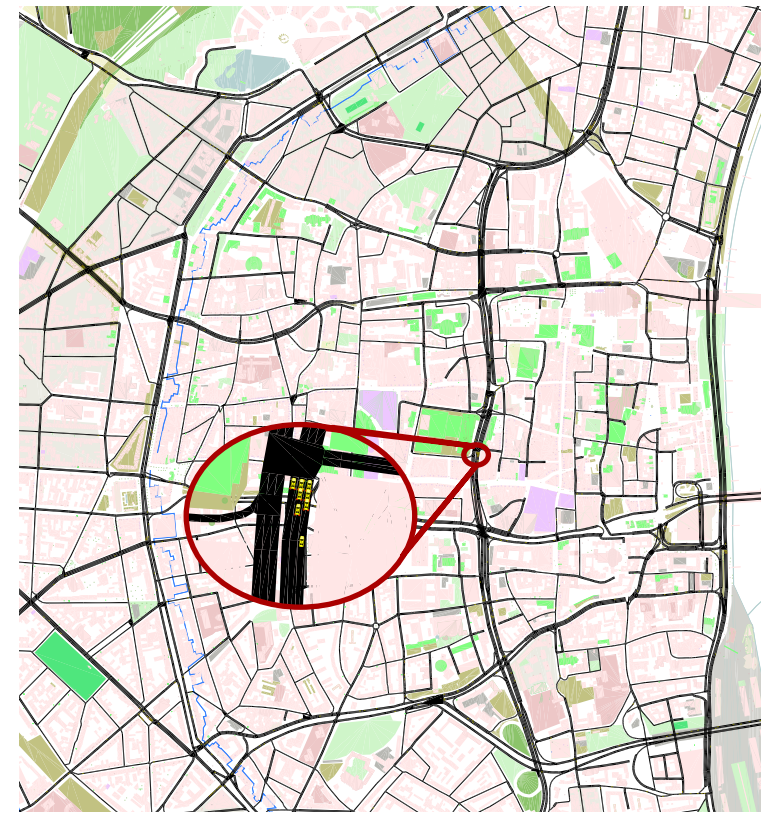

Figure 3. Cologne simulation scenario

Table I

SIMULATION PARAMETERS

\begin{tabular}{lc}
\hline Parameter & Value \\
\hline Manhattan Grid target area & $620 \mathrm{~m} \times 530 \mathrm{~m}$ \\
Cologne target area & $2.16 \mathrm{~km} \times 2.43 \mathrm{~km}$ \\
Manhattan Grid density (veh/km/lane) & 33 \\
Cologne density (veh/km ${ }^{2}$ ) & 95 \\
Inter-Vehicle Communication (IVC) technology & IEEE $802.11 \mathrm{p}$ \\
IVC maximal transmit power (mW) & 20 \\
DSRC beacon frequency (ms) & $10,20,30,50,100$ \\
DSRC bitrate (Mbps) & 3 \\
Payload length (B) & 1000 \\
\hline
\end{tabular}

Figure 3), which covers a region of $400 \mathrm{~km}^{2}$ in the city of Cologne, Germany, and reproduces with a high level of realism the vehicular traffic for a period of $24 \mathrm{~h}$. For our simulations, we delimited a $2.16 \mathrm{~km} \times 2.43 \mathrm{~km}$ target area situated in the center of Cologne city, represented in Figure 3.

The vehicular mobility is simulated with SUMO [20], a micro-mobility road traffic simulator, while the vehicular networking part is simulated with the well-known framework composed of OMNeT++ [21] and Veins [22]. Vehicles are following the Krauss vehicular mobility model and the random trips traffic flow origin-destination model. The mobility is generated with a fringe factor equal to 10 , meaning that it is 10 times more likely that the trips will start/end at the fringe of the simulated scenario. In this way, we model our vehicular traffic to start and end outside of the target area.

We assume that all vehicles have DSRC technology on-board. IEEE 802.11p parameters are considered for MAC and PHY. Two attenuation models are used: the free-space path loss with $\alpha=2$, and the simple obstacle shadowing [23] to model the impact of buildings on signal propagation. The main simulation parameters can be found in Table I. 
At the application level, a simple beacon exchange mechanism is implemented, where every vehicle, periodically with period $T_{\mathrm{msg}}$ and independently, broadcasts a beacon message containing basic information, like identification, position, velocity, timestamp, etc. $T_{\mathrm{msg}}$ is a global parameter known to all vehicles. Also, every vehicle maintains a Local Data Base (LDB) (i.e., simulating an LDM) where the information from the incoming beacon messages is stored. In a separate data structure, the last arrival times for every neighboring vehicle are saved, so as to be able to compute the time between two consecutive receptions of a beacon message from the same neighbor.

In the simulation vehicles enter the RoI in the considered urban map, roam in the RoI, then eventually they leave it. We focus on an observation time interval $\mathcal{I}=\left[t_{0}-\Delta / 2, t_{0}+\Delta / 2\right]$, where $t_{0}$ is a generic time of the statistical equilibrium regime of the simulation. Each vehicle collects messages coming from its neighbors. Let $\mathcal{K}_{i j}$ be the set of indices of messages originated by node $i$ and received by node $j$ during the interval $\mathcal{I}$. Let further $\Delta t_{i j}(r)$ be the time interval spanning between the reception of the $r$ and the $(r+1)$-th message from $i$ at $j$, for $r \in \mathcal{K}_{i j}$. Then, the estimate of the average AoI of messages from $j$ at node $i$ is given by (see Figure 1)

$$
\hat{H}_{i j}=\frac{\frac{1}{2} \sum_{r \in \mathcal{K}_{i j}} \Delta t_{i j}^{2}(r)}{\sum_{r \in \mathcal{K}_{i j}} \Delta t_{i j}(r)}
$$

Let $\Delta_{i j} \equiv \sum_{r \in \mathcal{K}_{i j}} \Delta t_{i j}(r)$. The overall average of the AoI can be obtained by averaging the $\hat{H}_{i j}$ 's, weighted by the fraction of the observation time when the messages have been collected, namely

$$
\hat{H}=\sum_{i=1}^{N(\mathcal{I})} \sum_{j=1}^{N(\mathcal{I})} \frac{\Delta_{i j}}{\Delta} \hat{H}_{i j}
$$

where $N(\mathcal{I})$ is the number of vehicle seen roaming in the RoI during the a time interval $\mathcal{I}$ in the statistical equilibrium regime. Putting together Equations (13) and (14), we can write

$$
\hat{H}=\frac{1}{2 \Delta} \sum_{k \in \mathcal{M}(\mathcal{I})} \Delta t_{k}^{2}
$$

where $\mathcal{M}(\mathcal{I})$ is the set of all messages received by some vehicle during the interval $\mathcal{I}$.

\section{NUMERICAL RESULTS}

For the validation against simulations, the analytical model takes in input the network connectivity graph based on the carrier sensing, which we obtain from simulations by taking snapshots over time of the vehicular network. In particular, we obtain the adjacency matrix $\mathbf{A}$ from a short simulation of the beacon exchange process, where every vehicle is sending in broadcast a beacon every $T_{\mathrm{msg}}$. This allows every vehicle to build its own LDB, which we then use to build the network connectivity graph (i.e., the adjacency matrix $\mathbf{A}$ ). By doing so, we make sure that $\mathbf{A}$ is obtained by accounting for the radio channel model built into the simulator and described in

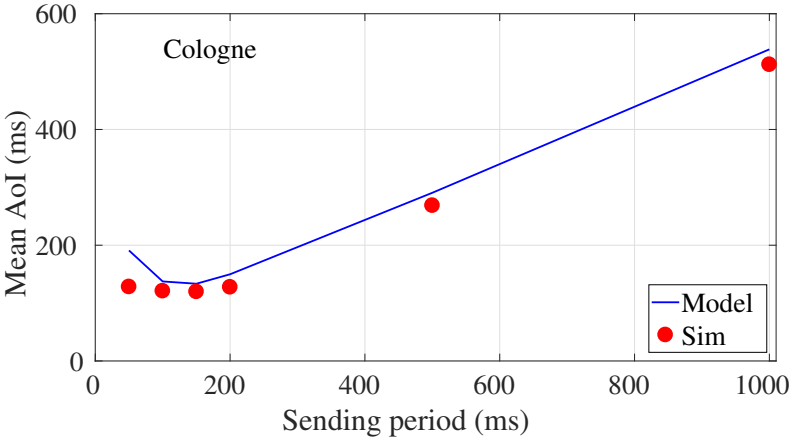

Figure 4. Cologne: mean Age-of-Information with respect to sending period analytical model (Model) vs simulation (Sim)

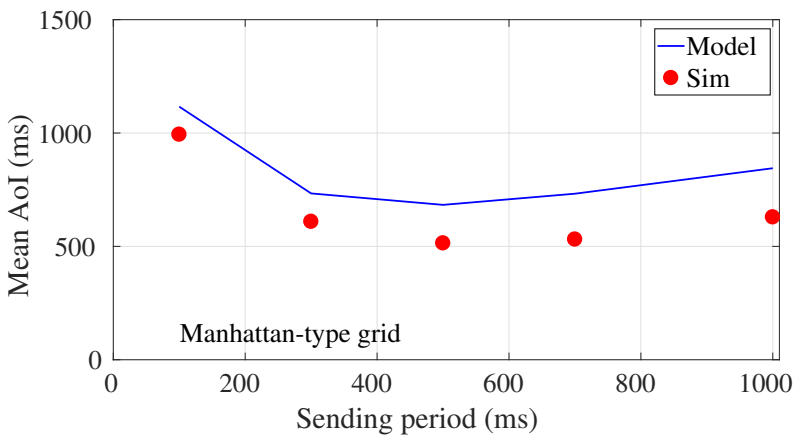

Figure 5. Manhattan Grid: mean Age-of-Information with respect to sending period - analytical model (Model) vs simulation (Sim)

Section IV. The values of AoI obtained from simulations are computed according to the Equation (15).

In Figures 4 and 5 we show the average AoI when varying the sending period for the Cologne and Manhattan Grid scenarios respectively. We can see that the results obtained with the analytical model are close to the simulation results, meaning that the proposed model is able to capture and approximate quite well how the average AoI changes with the sending period. The model yields a less accurate upper bound for the Manhattan Grid scenario and for the lowest value of the sending period in case of the Cologne scenario. In both cases, the model provides an upper bound of the actual performance anyway. An important observation is that the model captures the optimal level of the message sending interval, which is around $150 \mathrm{~ms}$ for Cologne and $500 \mathrm{~ms}$ for Manhattan Grid. When we depart from these optimal levels, the AoI starts increasing. Moreover, even at the optimal sending interval, the AoI is quite higher than the ideal level $T_{\mathrm{msg}} / 2$.

This phenomenon can be explained by the fact that for such low intervals the channel is highly congested, as can be seen from Figure 6, which leads to a higher message loss ratio and to the fact that a backlogged node has to wait for a much longer time to sense the channel idle. Message loss impacts strongly the AoI, leading to variable and stochastically high gaps between received updates. On the other side, when the sending period grows, the contention on the wireless channel is relieved, but then the AoI starts increasing again because 


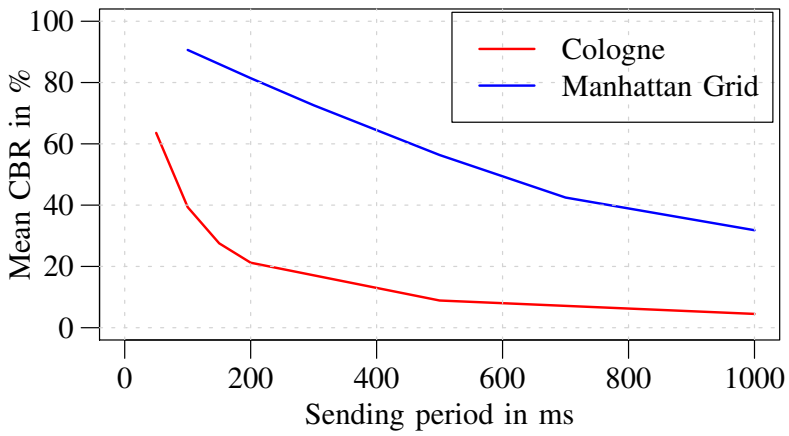

Figure 6. Mean channel busy ratio (CBR) with respect to sending period

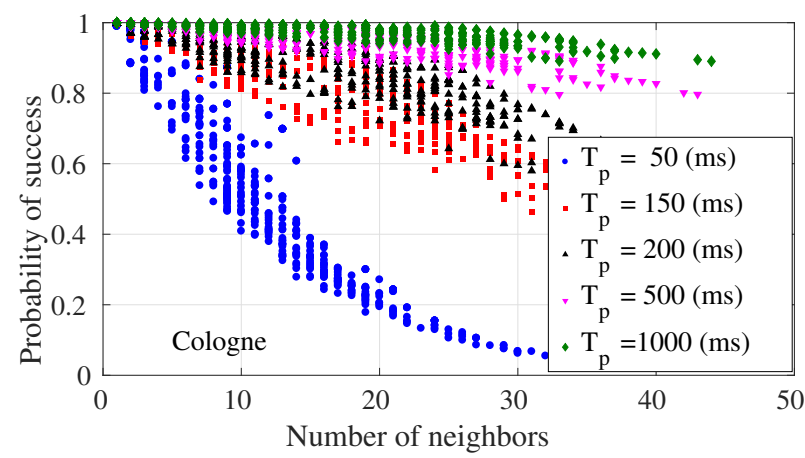

Figure 7. Probability of successful message delivery with respect to the number of DSRC neighbors for different sending periods

of the large time between the sending of successive updates. Figure 6 also shows that the Manhattan Grid scenario is much more congested than Cologne.

Figure 7 is a scatter plot of the average probability of successful message delivery for node $i$, namely $\bar{P}_{s}(i)=$ $\sum_{j} P_{s}(i, j) a_{i j} / \nu_{i}$, as a function of the number of neighbors $\nu_{i}$. It is apparent that the average success probability depends on how crowded the node neighborhood is, which is directly related to the air interface congestion. For longer message sending periods (e.g., $T_{\mathrm{msg}}=1000 \mathrm{~ms}$ ) the dependence of $\bar{P}_{s}(i)$ versus $\nu_{i}$ is weak, whereas a wide range of levels of $\bar{P}_{s}(i)$ can be observed for shorter periods. This points out that the local effectiveness of message refreshing becomes critically dependent on the local vehicle density as the message sending period is decreased, i.e., locally different performance can be experienced by vehicles. This finding is consistent with the observations in [24] and [25], where the authors propose adaptive beaconing solutions to cope with variable vehicle density.

Figure 8 shows the (net) average throughput of a node, that is to say the average amount of data delivered by a node to its neighboring nodes successfully. This is simply $\bar{P}_{s}(i) / \mathrm{E}\left[Y_{i}\right]$. It is apparent that the more the neighbors of a node, the less the amount of throughput that the node can sustain. This is consistent with the intuition that in crowded network spots the high level of contention hinders the possibility of delivering update messages to neighboring nodes. This

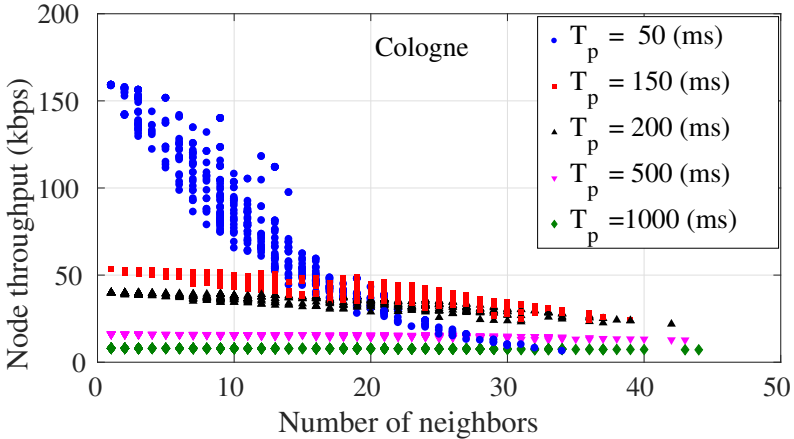

Figure 8. Average net throughput of a node with respect to the number of DSRC neighbors for different sending periods

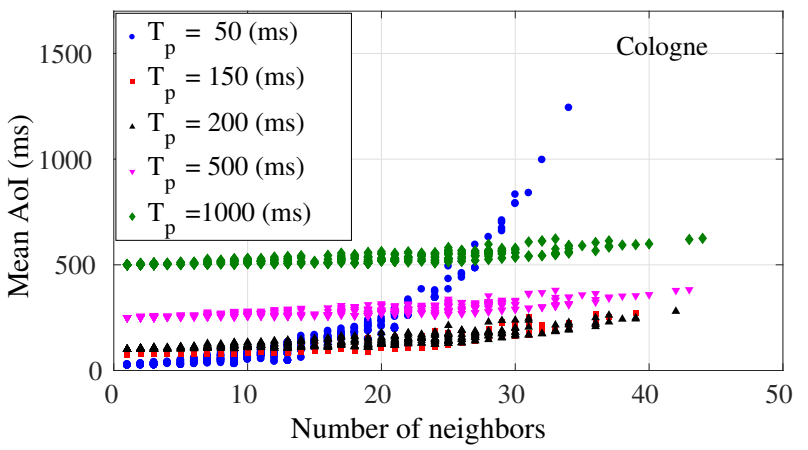

Figure 9. Average AoI of nodes with respect to the number of DSRC neighbors for different sending periods

is strongly amplified according to the level of $T_{\mathrm{msg}}$. As the message sending frequency $1 / T_{\mathrm{msg}}$ grows, the throughput levels change from essentially constant with the number of neighbors to extremely sensitive to the number of neighbors (e.g., see $T_{\mathrm{msg}}=50 \mathrm{~ms}$, where the throughput drops by more than one order of magnitude from low to high number of neighbors). Increasing the message sending frequency triggers an increasing level of unfairness in the message throughput performance experienced by nodes as a function of the local vehicle density, The best comprise is achieved around a message sending period between 150 and $200 \mathrm{~ms}$.

Finally, Figure 9 shows the average AoI per node $\mathrm{E}\left[H_{i}\right]$ against the number of neighboring nodes. In general, $\mathrm{E}\left[H_{i}\right]$ grows with the number of neighbors. While the dependence is weak for large levels of $T_{\mathrm{msg}}$, it gets dramatic for the lowest considered level of the message sending period, where $\mathrm{E}\left[H_{i}\right]$ spans two orders of magnitude. Thus, maintaining balanced performance among different nodes requires avoiding too small levels of $T_{\mathrm{msg}}$. However, large levels of $T_{\mathrm{msg}}$ entail a large AoI anyway, due to the sporadic refresh of information. The best compromise between 'stable' performance of nodes, irrespective of the local vehicle density and small AoI levels, is achieved for $T_{\mathrm{msg}}$ ranging between 150 and $200 \mathrm{~ms}$.

A spatial representation of the per-node AoI $\mathrm{E}\left[H_{i}\right]$ is shown in Figure 10. Dots correspond to vehicles and are scattered according to their registered position at the observation time in the simulation. The average per-node AoI calculated for 


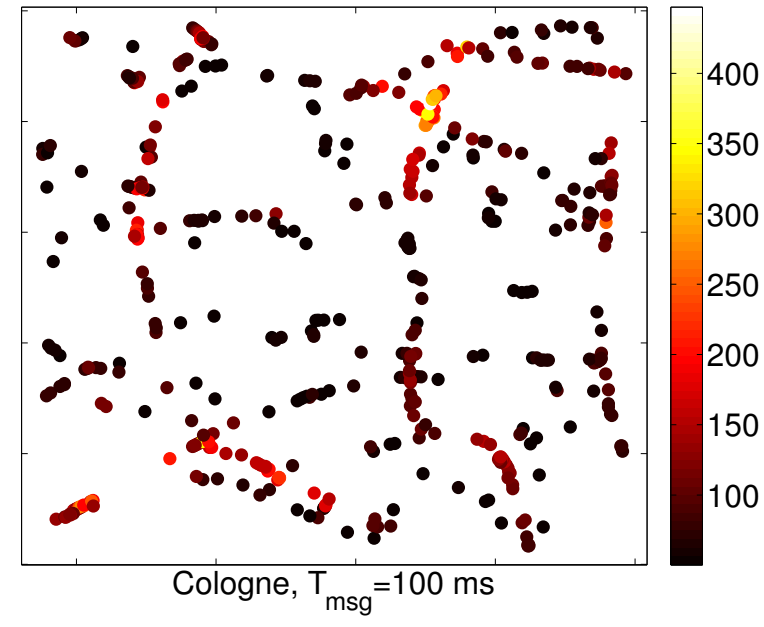

Figure 10. Average AoI of nodes represented with a heat colormap (scale in milliseconds). Dots correspond to vehicles, scattered according to their respective positions at a given time of the simulation.

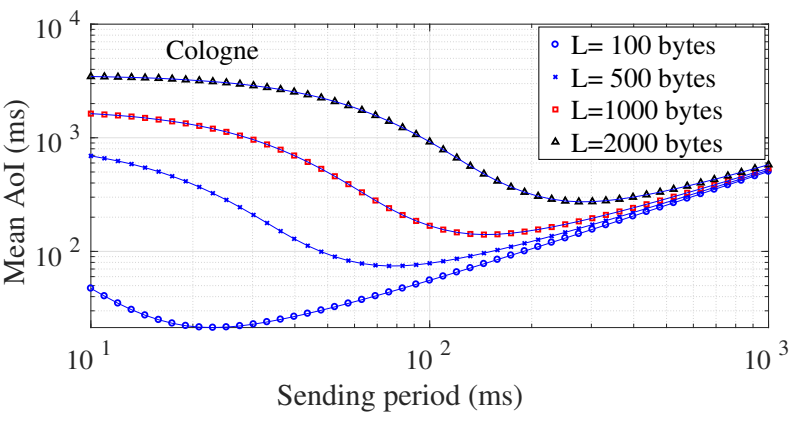

Figure 11. Mean Age-of-Information with respect to sending period for different packet lengths

each node according to the model is shown by using a heat colormap labelled with AoI levels in milliseconds. From the layout of the nodes, it is apparent that hot spots (i.e., zones where the AoI level experienced by nodes is high) are located mainly at road crossings.

The model can be used to gain insight into the effect of $T_{\mathrm{msg}}$ as well as other system parameters. Figure 11 shows the mean Age-of-Information with respect to sending period for different packet lengths $L$. The best operating point, i.e., the one minimizing the AoI, grows substantially as the packet length is increased. It is about $20 \mathrm{~ms}$ for $L=100 \mathrm{~B}$, while for $L=2000 \mathrm{~B}$ the minimum AoI is attained around $T_{\mathrm{msg}} \sim$ $500 \mathrm{~ms}$. As the message sending frequency increases, the AoI performance tends to saturate. This corresponds to the fact that the DSRC air interface gets saturated and the MAC level performance becomes essentially independent of the message sending period (i.e., nodes always have a new message to send).

\section{CONCLUSION}

In this article we define an analytical model to evaluate the Age-of-Information of a VANET, defined as the mean age of the latest update received by neighboring nodes, given the network connectivity graph of the vehicles. We validate the proposed solution by comparing it with realistic simulations of an urban area representing an area of the Cologne city and a generic Manhattan Grid scenario. Our results show that the analytical model is able to capture and approximate quite well how the average AoI changes with respect to the beacon sending frequency. It points out that an unfairness problem exists, i.e., AoI is directly related to vehicle density. Increasing the message sending period is beneficial for those vehicles that move in sparser zones, whereas it can degrade AoI strongly if used for vehicles in crowded areas. The analytical model can be used to set the sending period so as to minimize the AoI metric. It could also be used to guide the development of adaptive beaconing algorithms.

Further work could address the limitations of the analytical model, i.e., its inaccuracy as the mean vehicle density grows. The key point that calls for more investigation is capturing the effect of partial sensing, which is done in a simple, yet coarse way in our model by exploiting the clustering coefficient. A second point is to understand whether the relevant node connectivity information for the evaluation of the AoI can be reduced to some global property of the carrier sensing graph, rather than to the detailed adjacency matrix.

\section{REFERENCES}

[1] J. B. Kenney, "Dedicated Short-Range Communications (DSRC) Standards in the United States," Proceedings of the IEEE, vol. 99, no. 7, pp. 1162-1182, Jul. 2011.

[2] European Telecommunications Standards Institute, "Intelligent Transport Systems (ITS); Access layer specification for Intelligent Transport Systems operating in the $5 \mathrm{GHz}$ frequency band," ETSI, EN 302663 V1.2.1, Jul. 2013.

[3] — - "Intelligent Transport Systems (ITS); Vehicular Communications; Basic Set of Applications; Part 2: Specification of Cooperative Awareness Basic Service,” ETSI, Tech. Rep. 302 637-2 V1.3.2, Nov. 2014.

[4] — "Intelligent Transport Systems (ITS); Vehicular Communications; Basic Set of Applications; Part 3: Specification of Decentralized Environmental Notification Basic Service," ETSI, Tech. Rep. 302 637-3 V1.2.1, Sep. 2014.

[5] N. M. Freris, H. Kowshik, and P. R. Kumar, "Fundamentals of Large Sensor Networks: Connectivity, Capacity, Clocks, and Computation," Proceedings of the IEEE, vol. 98, no. 11, pp. 1828-1846, Nov. 2010.

[6] H. Zhang, Z. Zhang, and H. Dai, "Gossip-Based Information Spreading in Mobile Networks," IEEE Transactions on Wireless Communications, vol. 12, no. 11, pp. 5918-5928, Nov. 2013.

[7] J. Liu, S. Mou, A. S. Morse, B. D. O. Anderson, and C. Yu, "Deterministic Gossiping," Proceedings of the IEEE, vol. 99, no. 9, pp. 1505-1524, Sep. 2011.

[8] M. Saeednia and M. Menendez, "A Consensus-Based Algorithm for Truck Platooning," IEEE Transactions on Intelligent Transportation Systems, vol. 18, no. 2, pp. 404-415, Feb. 2017.

[9] J. He, P. Cheng, L. Shi, J. Chen, and Y. Sun, "Time Synchronization in WSNs: A Maximum-Value-Based Consensus Approach," IEEE Transactions on Automatic Control, vol. 59, no. 3, pp. 660-675, Mar. 2014.

[10] N. Kumar, S. Misra, M. S. Obaidat, J. J.P. C. Rodrigues, and B. Pati, "Networks of learning automata for the vehicular environment: a performance analysis study," IEEE Wireless Communications, vol. 21, no. 6, pp. 41-47, Dec. 2014.

[11] G. Scutari, S. Barbarossa, and L. Pescosolido, "Distributed Decision Through Self-Synchronizing Sensor Networks in the Presence of Propagation Delays and Asymmetric Channels," IEEE Transactions on Signal Processing, vol. 56, no. 4, pp. 1667-1684, Apr. 2008. 
[12] S. Kaul, R. Yates, and M. Gruteser, "Real-time status: How often should one update?" In Proceedings IEEE INFOCOM 2012, Mar. 2012 pp. 2731-2735.

[13] S. Kaul, M. Gruteser, V. Rai, and J. Kenney, "Minimizing age of information in vehicular networks," in 8th Annual IEEE Communications Society Conference on Sensor, Mesh and Ad Hoc Communications and Networks, Jun. 2011, pp. 350-358.

[14] A. Franco, E. Fitzgerald, B. Landfeldt, N. Pappas, and V. Angelakis, "LUPMAC: A cross-layer MAC technique to improve the age of information over dense WLANs," in 23rd International Conference on Telecommunications (ICT), May 2016, pp. 1-6.

[15] G. Bianchi, "Performance analysis of the IEEE 802.11 distributed coordination function," IEEE Journal on Selected Areas in Communications, vol. 18, no. 3, pp. 535-547, Mar. 2000.

[16] S. C. Liew, C. H. Kai, H. C. Leung, and P. Wong, "Back-of-theEnvelope Computation of Throughput Distributions in CSMA Wireless Networks," IEEE Transactions on Mobile Computing, vol. 9, no. 9, pp. 1319-1331, Sep. 2010.

[17] R. Laufer and L. Kleinrock, "The Capacity of Wireless CSMA/CA Networks," IEEE/ACM Transactions on Networking, vol. 24, no. 3, pp. 1518-1532, Jun. 2016

[18] European Telecommunications Standards Institute, "Intelligent Transport Systems (ITS); Vehicular Communications; Basic Set of Applications; Local Dynamic Map (LDM)," ETSI, EN 302895 V1.1.1, Sep. 2014

[19] S. Uppoor, O. Trullols-Cruces, M. Fiore, and J. M. Barcelo-Ordinas, "Generation and Analysis of a Large-Scale Urban Vehicular Mobility
Dataset,' IEEE Transactions on Mobile Computing, vol. 13, no. 5, pp. 1061-1075, 2014

[20] D. Krajzewicz, G. Hertkorn, C. Rössel, and P. Wagner, "SUMO (Simulation of Urban MObility); An Open-source Traffic Simulation,' in 4th Middle East Symposium on Simulation and Modelling (MESM 2002), Sharjah, United Arab Emirates, Sep. 2002, pp. 183-187.

[21] A. Varga and R. Hornig, "An overview of the OMNeT++ simulation environment," in 1st ACM/ICST International Conference on Simulation Tools and Techniques for Communications, Networks and Systems (SIMUTools 2008), Marseille, France: ACM, Mar. 2008.

[22] C. Sommer, R. German, and F. Dressler, "Bidirectionally Coupled Network and Road Traffic Simulation for Improved IVC Analysis," IEEE Transactions on Mobile Computing, vol. 10, no. 1, pp. 3-15, Jan. 2011.

[23] C. Sommer, D. Eckhoff, R. German, and F. Dressler, "A Computationally Inexpensive Empirical Model of IEEE 802.11p Radio Shadowing in Urban Environments," in 8th IEEE/IFIP Conference on Wireless On demand Network Systems and Services (WONS 2011), Bardonecchia, Italy: IEEE, Jan. 2011, pp. 84-90.

[24] C. Sommer, O. K. Tonguz, and F. Dressler, "Adaptive Beaconing for Delay-Sensitive and Congestion-Aware Traffic Information Systems," in 2nd IEEE Vehicular Networking Conference (VNC 2010), Jersey City, NJ: IEEE, 2010, pp. 1-8.

[25] C. Sommer, S. Joerer, M. Segata, O. K. Tonguz, R. Lo Cigno, and F. Dressler, "How Shadowing Hurts Vehicular Communications and How Dynamic Beaconing Can Help," IEEE Transactions on Mobile Computing, vol. 14, no. 7, pp. 1411-1421, 2015. 